\title{
Modeling of microwave devices with space mapping and radial basis functions
}

\author{
Slawomir Koziel $^{1, *, \dagger}$ and John W. Bandler ${ }^{1,2}$ \\ ${ }^{1}$ Simulation Optimization Systems Research Laboratory, Department of Electrical and Computer Engineering, \\ McMaster University, Hamilton, ON, Canada L8S 4K1 \\ ${ }^{2}$ Bandler Corporation, Dundas, ON, Canada L9H $5 E 7$
}

\begin{abstract}
SUMMARY
We review recent developments in space mapping techniques for modeling of microwave devices. We present a surrogate modeling methodology that utilizes space mapping combined with radial basis function interpolation. The method has advantages both over the standard space mapping modeling methodology and the recently published space mapping modeling with variable weight coefficients. In particular, it provides accuracy comparable or better than the latter method and computational efficiency as good as the standard space mapping modeling procedure. A comparison between the space mapping modeling methodologies as well as application examples of optimization and statistical analysis of microwave structures is presented. Copyright (C) 2007 John Wiley \& Sons, Ltd.
\end{abstract}

Received 29 March 2007; Accepted 18 June 2007

KEY WORDS: computer-aided design (CAD); EM modeling; space mapping; surrogate modeling; radial basis functions; engineering optimization

\section{INTRODUCTION}

Statistical analysis and yield optimization, crucial for manufacturability-driven designs in a time-to-market development environment, demand accurate and fast models. Full-wave EM simulations of microwave structures offer accuracy at the cost of CPU effort. High CPU cost is undesirable from the point of view of direct statistical analysis and design. The space mapping concept $[1-8,27]$ addresses this issue.

\footnotetext{
*Correspondence to: Slawomir Koziel, Simulation Optimization Systems Research Laboratory, Department of Electrical and Computer Engineering, McMaster University, Hamilton, ON, Canada L8S 4K1.

†E-mail: koziels@mcmaster.ca

Contract/grant sponsor: Natural Sciences and Engineering Research Council of Canada; contract/grant numbers: OGP0007239, STPGP336760
} 
Space mapping assumes the existence of 'fine' and 'coarse' models. The 'fine' model may be a high fidelity CPU-intensive EM simulator, undesirable for direct statistical analysis and design. The 'coarse' model can be a simplified representation such as an equivalent circuit with empirical formulas. Space mapping modeling [9-15] and neuro-space-mapping modeling [16-18] exploit the speed of the coarse model and the accuracy of the fine model to develop fast, accurate enhanced models (surrogates) valid over a wide range of parameter values. The main factor that differentiates space mapping from other surrogate-based modeling methodologies (e.g. [19-24]) is the use of a physics-based coarse model, which facilitates obtaining good modeling accuracy for a small amount of fine model data.

The standard SM modeling approach is based on setting up the surrogate model using a small amount of fine model data (usually a so-called star distribution: $2 n+1$ points, where $n$ is the number of design variables) and performing extraction of the mapping parameters over the whole set of these data $[10,11]$. This simple methodology gives reasonable accuracy especially for low-dimensional problems. To further improve modeling performance, one needs to involve a larger amount of fine model information. Unfortunately, space mapping is not suited to handling a large amount of fine model data by itself, i.e. increasing the number of base points does not help if the number of surrogate model parameters remains unchanged.

A recently published space mapping modeling approach with variable weight coefficients $[13,14]$ was aimed at overcoming these limitations. It indeed provides better accuracy than the standard method, however, at the expense of significant increase of the evaluation time, which is due to a separate parameter extraction required for each evaluation of the surrogate model. This limits potential applications of the method.

A novel approach [15] combines the standard space mapping modeling methodology with radial basis function interpolation. This combination gives modeling accuracy comparable or better than the variable weight method [13] without compromising computational cost. Moreover, because of the underlying coarse model, modeling accuracy is substantially better than for radial basis function interpolation used directly.

In this paper, we present a brief description and comparison of space mapping modeling methodologies. We also provide application examples of space mapping modeling techniques for optimization and statistical analysis of microwave structures. Numerical results confirm that a combination of space mapping and radial basis functions is the best space-mapping-based modeling methodology thus far, both with respect to accuracy and computational efficiency.

\section{SPACE MAPPING MODELING METHODOLOGIES}

In this section, we present three space-mapping-based modeling methodologies. We will use the following notation. Let $\mathbf{R}_{f}: X_{f} \rightarrow R^{m}$ and $\mathbf{R}_{c}: X_{c} \rightarrow R^{m}$ denote the fine and coarse model response vectors, where $X_{f} \subseteq R^{n}$ and $X_{c} \subseteq R^{n}$ are design variable domains of the fine and coarse models, respectively. For example, $\mathbf{R}_{f}(\mathbf{x})$ and $\mathbf{R}_{c}(\mathbf{x})$ may represent the magnitude of a transfer function of a microwave filter at $m$ chosen frequencies. We denote by $X_{R} \subseteq X_{f}$ the region of interest in which we want enhanced matching between the surrogate and the fine model. We assume that $X_{R}$ is an $n$-dimensional interval in $R^{n}$ centered at reference point $\mathbf{x}^{0}=\left[x_{0.1} \ldots x_{0 . n}\right]^{\mathrm{T}} \in R^{n}$ :

$$
X_{R}=\left[\mathbf{x}^{0}-\boldsymbol{\delta}, \mathbf{x}^{0}+\boldsymbol{\delta}\right]=\left[x_{0.1}-\delta_{1}, x_{0.1}+\delta_{1}\right] \times \cdots \times\left[x_{0 . n}-\delta_{n}, x_{0 . n}+\delta_{n}\right]
$$


where $\boldsymbol{\delta}=\left[\delta_{1} \ldots \delta_{n}\right]^{\mathrm{T}}$ determines the size of $X_{R}$. We use $X_{R}\left(\mathbf{x}^{0}, \boldsymbol{\delta}\right)$ to denote the region of interest defined by $\mathbf{x}^{0}$ and $\boldsymbol{\delta}$. Suppose we have the base set $X_{B}=\left\{\mathbf{x}^{1}, \mathbf{x}^{2}, \ldots, \mathbf{x}^{N}\right\} \subset X_{R}\left(\mathbf{x}^{0}, \boldsymbol{\delta}\right)$, where $N$ is the number of base points, such that the fine model response is known at all points $\mathbf{x}^{j}, j=1,2, \ldots, N$. In general, we do not assume any particular location of these base points.

\subsection{Standard space mapping modeling}

Standard space mapping model (SM-Standard) is a generic surrogate model $\mathbf{R}_{s}: X_{f} \times M_{m \times m} \times$ $M_{n \times n} \times M_{n \times 1} \rightarrow R^{m}$ defined as [10]

$$
\overline{\mathbf{R}}_{s}(\mathbf{x}, \mathbf{A}, \mathbf{B}, \mathbf{c})=\mathbf{A} \cdot \mathbf{R}_{c}(\mathbf{B} \cdot \mathbf{x}+\mathbf{c})
$$

with matrices $\mathbf{A}=\operatorname{diag}\left\{a_{1}, \ldots, a_{m}\right\}, \mathbf{B} \in M_{n \times n}$, and $\mathbf{c} \in M_{n \times 1}\left(M_{k \times l}\right.$ denotes the set of $k \times l$ real matrices) found using the parameter extraction process

$$
(\mathbf{A}, \mathbf{B}, \mathbf{c})=\arg \min _{(\boldsymbol{\alpha}, \boldsymbol{\beta}, \boldsymbol{\eta})} \sum_{k=1}^{N}\left\|\mathbf{R}_{f}\left(\mathbf{x}^{k}\right)-\mathbf{R}_{s}\left(\mathbf{x}^{k}, \boldsymbol{\alpha}, \boldsymbol{\beta}, \boldsymbol{\eta}\right)\right\|
$$

Note that $\mathbf{R}_{s}$ is a coarse model composed with linear transformations. Apart from model (2), (3), an optional frequency scaling can be implemented that works in such a way that the coarse model is evaluated at a different frequency than the fine model using the transformation: $\omega \rightarrow$ $f_{0}+f_{1} \omega$, where $\mathbf{F}=\left[f_{0} f_{1}\right] \in R^{2}$ is obtained together with matrices $\mathbf{A}, \mathbf{B}$, and $\mathbf{c}$ using a parameter extraction process similar to (3). More general space mapping surrogate models can be found, e.g. in $[3,10]$.

The standard space mapping surrogate model is very simple and fast, because once the space mapping parameters are established, model evaluation cost is roughly the same as the evaluation cost of the coarse model, which is assumed to be much cheaper than the fine model. The limitation of this model is that linear mappings such as (2) may not be able to provide sufficient accuracy. Moreover, including more base points has little effect on the model's accuracy because of the finite number of model parameters, and also because the parameters are extracted in one shot for the whole region of interest.

\subsection{Space mapping modeling with variable weight coefficients}

Space mapping modeling with variable weight coefficients (SM-VWC) [13] overcomes the limitations of the standard model discussed above. This methodology uses the generic surrogate model (2), however, the model parameters are obtained in the following parameter extraction process:

$$
(\mathbf{A}, \mathbf{B}, \mathbf{c})=\arg \min _{(\boldsymbol{\alpha}, \boldsymbol{\beta}, \boldsymbol{\eta})} \sum_{k=1}^{N} w_{k}(\mathbf{x})\left\|\mathbf{R}_{f}\left(\mathbf{x}^{k}\right)-\mathbf{R}_{s}\left(\mathbf{x}^{k}, \boldsymbol{\alpha}, \boldsymbol{\beta}, \boldsymbol{\eta}\right)\right\|
$$

The weighting coefficients $w_{k}$ in (4) are functions of $\mathbf{x}$. They are calculated according to

$$
w_{k}=w_{k}(\mathbf{x} ; C, \gamma)=\frac{\exp \left(-\left\|\mathbf{x}-\mathbf{x}^{k}\right\|^{2} / C \cdot \gamma^{2}\right)}{\sum_{j=1}^{N} \exp \left(-\left\|\mathbf{x}-\mathbf{x}^{j}\right\|^{2} / C \cdot \gamma^{2}\right)}, \quad k=1,2, \ldots, N
$$


where $\mathbf{x}$ is the evaluation point, $\gamma=\gamma(\boldsymbol{\delta}, N)$ is a characteristic distance depending on the size of the region of interest and the number of base points

$$
\gamma(\boldsymbol{\delta}, N)=\frac{2}{n N^{1 / n}} \sum_{i=1}^{n} \delta_{i}
$$

If the base points are uniformly distributed in $X_{R}, \gamma=\gamma(\boldsymbol{\delta}, N)$ is just an average distance between neighboring points. Constant $C>0$ determines how fast the weighting coefficients decrease with increase of base-point distance from $\mathbf{x}$. Paper [13] contains a discussion on the implementation details of the method. In can be shown that this space mapping modeling methodology with variable weight coefficients can assure any required accuracy, provided that the base set is sufficiently dense, even if the number of model parameters is fixed [14]. The disadvantage of the method is the significant increase in the evaluation time, which is due to a separate parameter extraction process required for each evaluation of the surrogate model. This limits potential applications of the method.

\subsection{Space mapping modeling with radial basis functions}

The recent development in space mapping modeling is a combination of space mapping and radial basis functions (SM-RBF) [15]. In particular, on the top of the standard space mapping surrogate, a correction term is added that interpolates the difference between the fine model $\mathbf{R}_{f}$ and the standard space mapping surrogate $\mathbf{R}_{s}$ through radial basis functions $[25,26]$. Let $\mathbf{R}_{f}(\mathbf{x})=\left[R_{f .1}(\mathbf{x}) \ldots R_{f . m} \times\right.$ $(\mathbf{x})]^{\mathrm{T}}$ and $\mathbf{R}_{s}(\mathbf{x})=\left[R_{s .1}(\mathbf{x}) \ldots R_{s . m}(\mathbf{x})\right]^{\mathrm{T}}$. The radial basis function model $\tilde{\mathbf{R}}_{s}$ is defined as

$$
\tilde{\mathbf{R}}_{s}(\mathbf{x})=\left[\begin{array}{c}
\sum_{j=1}^{N} \lambda_{1 . j} \phi\left(\left\|\mathbf{x}-\mathbf{x}^{j}\right\| / \gamma\right) \\
\cdots \\
\sum_{j=1}^{N} \lambda_{m . j} \phi\left(\left\|\mathbf{x}-\mathbf{x}^{j}\right\| / \gamma\right)
\end{array}\right]
$$

where $\|$.$\| denotes the Euclidean norm. The parameters \lambda_{k . j}$ are calculated so that they satisfy

$$
\Phi \lambda_{k}=\mathbf{F}_{k}, \quad k=1,2, \ldots, m
$$

where $\lambda_{k}=\left[\begin{array}{llll}\lambda_{k .1} & \lambda_{k .2} & \ldots & \lambda_{k . N}\end{array}\right]^{\mathrm{T}}$,

$$
\mathbf{F}_{k}=\left[\begin{array}{c}
R_{f . k}\left(\mathbf{x}^{1}\right)-\bar{R}_{s . k}\left(\mathbf{x}^{1}\right) \\
\vdots \\
R_{f . k}\left(\mathbf{x}^{N}\right)-\bar{R}_{s . k}\left(\mathbf{x}^{N}\right)
\end{array}\right]
$$

and $\Phi$ is an $N \times N$ matrix with elements

$$
\Phi_{i j}=\phi\left(\left\|\mathbf{x}^{i}-\mathbf{x}^{j}\right\| / \gamma\right)
$$

with $\gamma=\gamma(\boldsymbol{\delta}, N)$ being a characteristic distance of the base set defined by (6). Parameter $\gamma$ is used in (4) as a normalization factor.

In this paper, we use a Gaussian basis function defined as

$$
\phi(r)=\mathrm{e}^{-c r^{2}}, \quad r \geqslant 0, c>0
$$

In the experiments presented in this paper, parameter $c$ is adjusted to minimize the generalization error calculated using the cross-validation method [22] ( $c$ was kept fixed and equal to 1 in [15]). Other choices of basis functions can be found in the literature [26]. 
The combined surrogate model $\overline{\mathbf{R}}_{s}: X_{R} \rightarrow R^{m}$ is defined as

$$
\overline{\mathbf{R}}_{s}(\mathbf{x})=\mathbf{R}_{s}(\mathbf{x})+\tilde{\mathbf{R}}_{s}(\mathbf{x})
$$

where $\mathbf{R}_{S}$ is a standard space mapping surrogate model, which is obtained using a subset of the base set $X_{B}$, e.g. the star distribution [10], however, in this paper, we use the whole $X_{B}$ to determine $\mathbf{R}_{s}$.

Once coefficients $\lambda$ are found, evaluation of (7) is fast, which means that the evaluation cost of model (10) is not significantly larger than the evaluation cost of the standard space mapping surrogate model (2). This is in contrast with the modeling technique [13], requiring a separate parameter extraction for each evaluation of the surrogate model, which involves a number of coarse model evaluations (typically hundreds or even thousands).

\section{COMPARISON OF MODELING METHODOLOGIES}

In this section, we compare the accuracy of the space mapping modeling techniques described in Section 2 using examples of microwave components and structures.

\subsection{Description of the test problems}

Problem 1: Microstrip right-angle bend [9]. The fine model, Figure 1(a), is analyzed by Sonnet's $\boldsymbol{e m}^{\mathrm{TM}}$ [28] using grid with a $0.5 \mathrm{mil} \times 0.5 \mathrm{mil}$ cell size. The coarse model, Figure $1(\mathrm{~b})$, is an equivalent circuit with parameters calculated from Kirschning et al. [29]. The design parameters are $\mathbf{x}=\left[\begin{array}{lll}W & H & \epsilon_{r}\end{array}\right]^{\mathrm{T}}$. The response vector consists of reflection coefficient $\left|S_{11}\right|$ in the frequency range $1-31 \mathrm{GHz}$. The reference point is $\mathbf{x}^{0}=\left[\begin{array}{lll}25 & 12 & 9\end{array}\right]^{\mathrm{T}}$, and the region size $\boldsymbol{\delta}=\left[\begin{array}{lll}6 & 4 & 1\end{array}\right]^{\mathrm{T}}$.

Problem 2: Second-order tapped-line microstrip filter [30] shown in Figure 2. The fine model is simulated in FEKO [31]. The coarse model, Figure 3, is the equivalent circuit model implemented in Agilent ADS [32]. The design parameters are $\mathbf{x}=\left[\begin{array}{ll}L_{1} & g\end{array}\right]^{\mathrm{T}}$. The response vector consists of transmission coefficient $\left|S_{21}\right|$ in the frequency range 3-7 GHz. The reference point is $\mathbf{x}^{0}=\left[\begin{array}{ll}6.977 & 0.060\end{array}\right]^{\mathrm{T}} \mathrm{mm}$ and the region size is $\boldsymbol{\delta}=\left[\begin{array}{ll}2 & 0.03\end{array}\right]^{\mathrm{T}} \mathrm{mm}$.

Problem 3: Bandstop microstrip filter with open stubs [33] shown in Figure 4. The fine model is simulated with Sonnet's $\boldsymbol{e m}$ [28] using high-resolution grid with a $0.2 \mathrm{mil} \times 1 \mathrm{mil}$ cell size. The coarse model, Figure 5, is the equivalent circuit model implemented in Agilent ADS [32]. The design parameters are $\mathbf{x}=\left[\begin{array}{lllll}W_{1} & W_{2} & L_{0} & L_{1} & L_{2}\end{array}\right]^{\mathrm{T}}$. The response vector consists

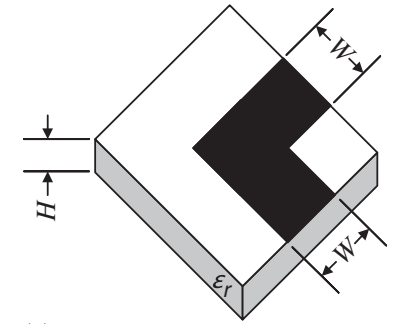

(a)



(b)

Figure 1. The microstrip right-angle bend: (a) the fine model and (b) the coarse model [9]. 


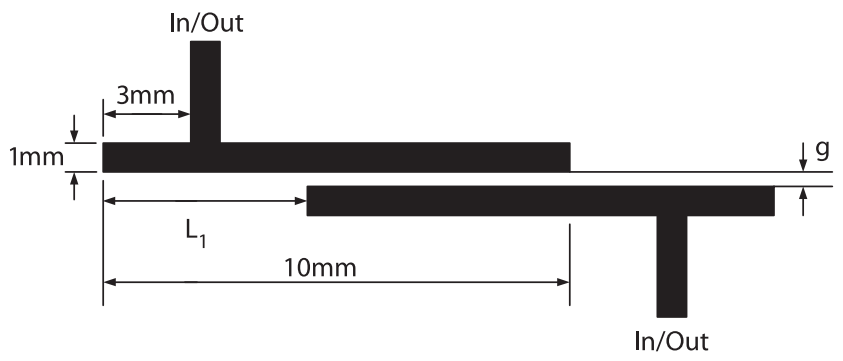

Figure 2. Geometry of the second-order tapped-line microstrip filter [30].

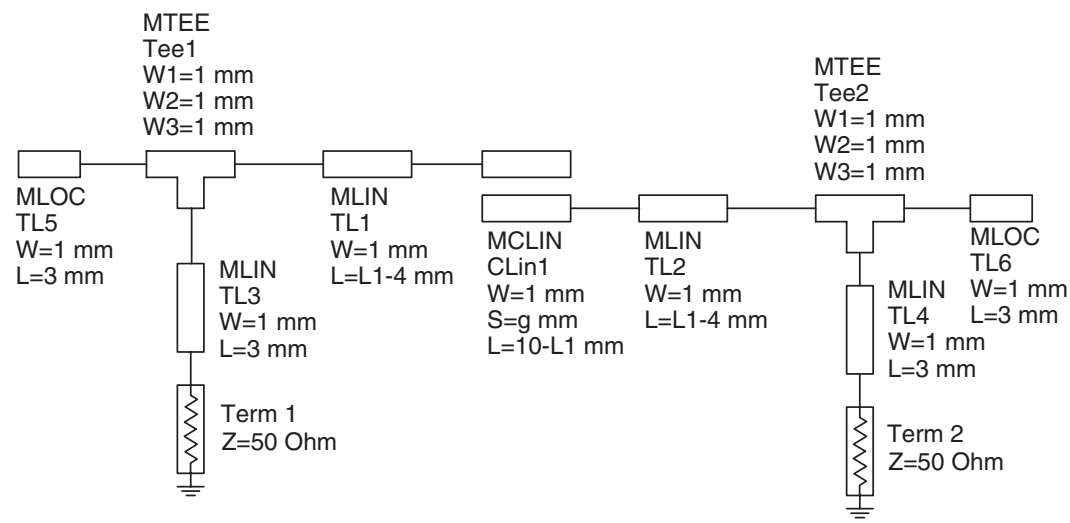

Figure 3. Coarse model of the second-order tapped-line filter (Agilent ADS).

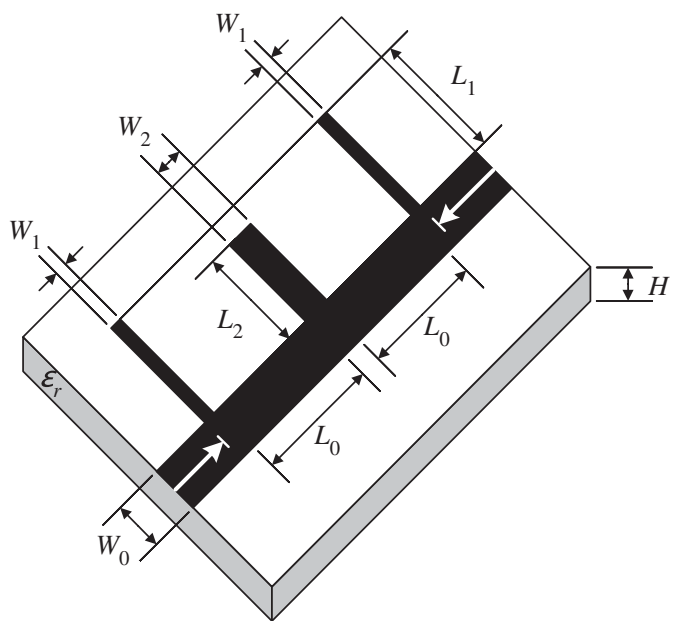

Figure 4. Bandstop microstrip filter with open stubs [33]. 
of transmission coefficient $\left|S_{21}\right|$ in the frequency range 5-15 GHz. The reference point is $\mathbf{x}^{0}=\left[\begin{array}{lllll}5.6 & 10.4 & 119.2 & 118 & 112\end{array}\right]^{\mathrm{T}}$ mil and the region size is $\boldsymbol{\delta}=\left[\begin{array}{lllll}0.4 & 0.4 & 2 & 2 & 2\end{array}\right]^{\mathrm{T}} \mathrm{mil}$.

\subsection{Experimental setup}

For each of the test problems, we performed a number of experiments using the following surrogate models: SM-Standard, SM-VWC, and SM-RBF. Table I gives details of the base sets used in our experiments. The base sets have growing numbers of points (and decreasing characteristic distances $\gamma$ ) in order to examine the dependence of the modeling error on the amount of fine model data used to create the model.

Accuracy was tested using 30 test points randomly distributed in the region of interest. The error measure used was the $l_{2}$ norm of the difference between the fine model response and the corresponding surrogate model response.

\subsection{Results and discussion}

Tables II-IV give numerical results (error statistics) for the models with the various base sets considered. Figures 6-8 show error plots (the modulus of the difference between the fine model and the corresponding surrogate model response versus frequency) for the SM-Standard and SM-RBF with base set $X_{B 3}$, respectively. Figures 9-11 show dependence of average modeling

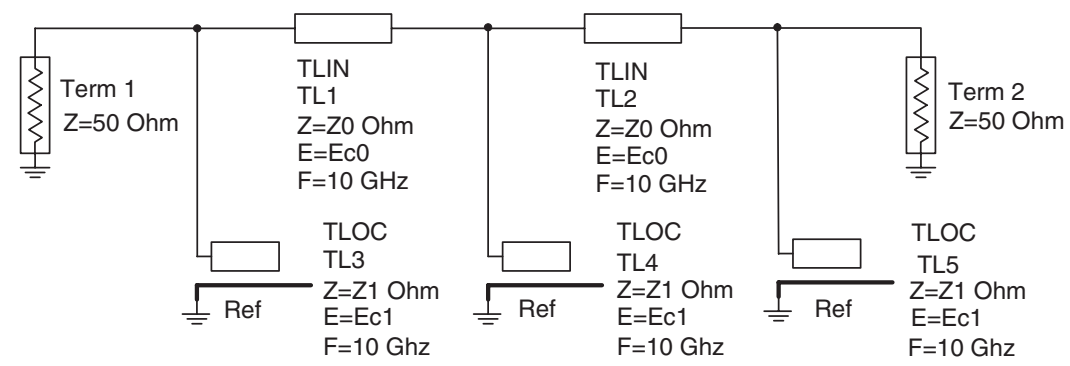

Figure 5. Coarse model of the bandstop microstrip filter with open stubs (Agilent ADS).

Table I. Base set data for test problems 1-3.

\begin{tabular}{lclcc}
\hline $\begin{array}{l}\text { Test } \\
\text { problem }\end{array}$ & $\begin{array}{c}\text { Base } \\
\text { set }\end{array}$ & \multicolumn{1}{c}{ Base set description } & $\begin{array}{c}\text { Number of base } \\
\text { points }\end{array}$ & $\gamma$ \\
\hline \multirow{4}{*}{1} & $X_{B 1}$ & Uniform mesh of density 2 + star distribution & 15 & 2.97 \\
& $X_{B 2}$ & Uniform mesh of density 3 & 27 & 2.44 \\
& $X_{B 3}$ & Uniform mesh of density 4 & 64 & 1.83 \\
2 & $X_{B 1}$ & Uniform mesh of density 3 & 9 & 0.68 \\
& $X_{B 2}$ & Uniform mesh of density 4 & 16 & 0.51 \\
& $X_{B 3}$ & Uniform mesh of density 5 & 25 & 0.41 \\
3 & $X_{B 1}$ & Star distribution & 11 & 1.68 \\
& $X_{B 2}$ & Uniform mesh of density 2 $\cup$ star distribution & 43 & 1.28 \\
& $X_{B 3}$ & Uniform mesh of density 2 $\cup$ star distribution & 73 & 1.15 \\
& & & & \\
\hline
\end{tabular}

*Thirty points randomly chosen out of points in the uniform mesh of density 3 but not belonging to $X_{B 2}$. 
Table II. Modeling results for test problem 1. Verification for 30 random test points.

\begin{tabular}{lcccc}
\hline Model & Base set & Average error & Maximum error & Standard deviation \\
\hline SM-Standard & & 0.0096 & 0.0219 & 0.0042 \\
SM-VWC & $X_{B 1}$ & 0.0063 & 0.0179 & 0.0037 \\
SM-RBF & & 0.0045 & 0.0096 & 0.0023 \\
& & & 0.0196 & 0.0037 \\
SM-Standard & & 0.0095 & 0.0179 & 0.0047 \\
SM-VWC & $X_{B 2}$ & 0.0086 & 0.0067 & 0.0016 \\
SM-RBF & & 0.0029 & 0.0184 & 0.0038 \\
& & 0.0084 & 0.0056 & 0.0012 \\
SM-Standard & $X_{B 3}$ & 0.0027 & 0.0013 & 0.0003 \\
SM-VWC & & 0.0005 &
\end{tabular}

Table III. Modeling results for test problem 2. Verification for 30 random test points.

\begin{tabular}{lcccc}
\hline Model & Base set & Average error & Maximum error & Standard deviation \\
\hline SM-Standard & & 2.57 & 5.52 & 1.30 \\
SM-VWC & $X_{B 1}$ & 2.47 & 7.33 & 1.85 \\
SM-RBF & & 1.40 & 2.77 & 0.84 \\
& & & 5.09 & 1.16 \\
SM-Standard & & 2.32 & 4.85 & 1.12 \\
SM-VWC & $X_{B 2}$ & 1.67 & 1.64 & 0.52 \\
SM-RBF & & 0.74 & 4.72 & 1.05 \\
& & 2.15 & 3.16 & 0.64 \\
SM-Standard & $X_{B 3}$ & 1.25 & 1.91 & 0.63 \\
SM-VWC & & 0.52 & &
\end{tabular}

Table IV. Modeling results for test problem 3. Verification for 30 random test points.

\begin{tabular}{lcccc}
\hline Model & Base set & Average error & Maximum error & Standard deviation \\
\hline SM-Standard & & 0.0389 & 0.0635 & 0.0084 \\
SM-VWC & $X_{B 1}$ & 0.0326 & 0.0483 & 0.0058 \\
SM-RBF & & 0.0055 & 0.0154 & 0.0038 \\
& & & 0.0581 & 0.0073 \\
SM-Standard & & 0.0403 & 0.0440 & 0.0051 \\
SM-VWC & $X_{B 2}$ & 0.0296 & 0.0108 & 0.0022 \\
SM-RBF & & 0.0024 & & \\
& & & 0.0599 & 0.0074 \\
SM-Standard & $X_{B 3}$ & 0.0404 & 0.0343 & 0.0061 \\
SM-VWC & & 0.0022 & 0.0110 & 0.0021 \\
SM-RBF & & & &
\end{tabular}

error on the characteristic distance $\gamma$. Table $\mathrm{V}$ presents the qualitative comparison of the surrogate model evaluation cost for all the considered methods.

The results show that the SM-RBF model-the combination of space mapping and RBF interpolation-outperforms both the standard SM model and the SM model with variable 

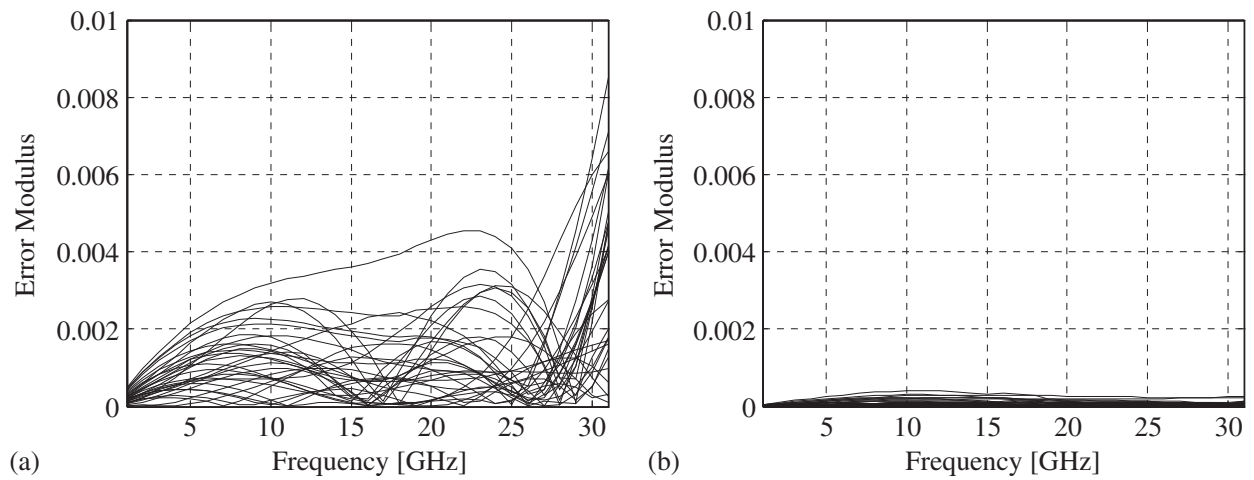

Figure 6. Test problem 1: error plots for (a) the SM-Standard and (b) SM-RBF surrogate models with base set $X_{B 3}$ (30 test points).
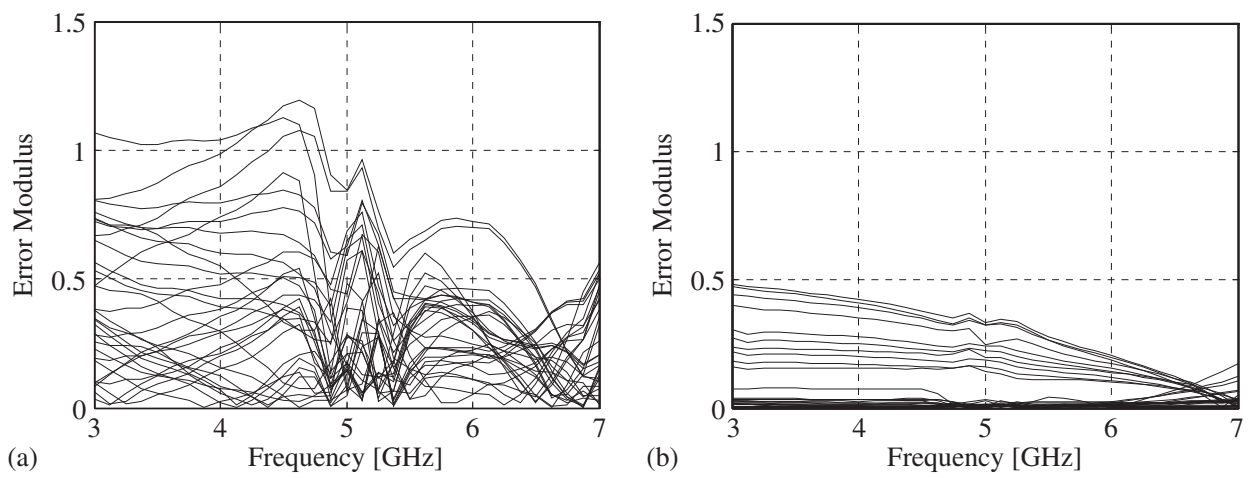

Figure 7. Test problem 2: error plots for (a) the SM-Standard and (b) SM-RBF surrogate models with base set $X_{B 3}$ (30 test points).
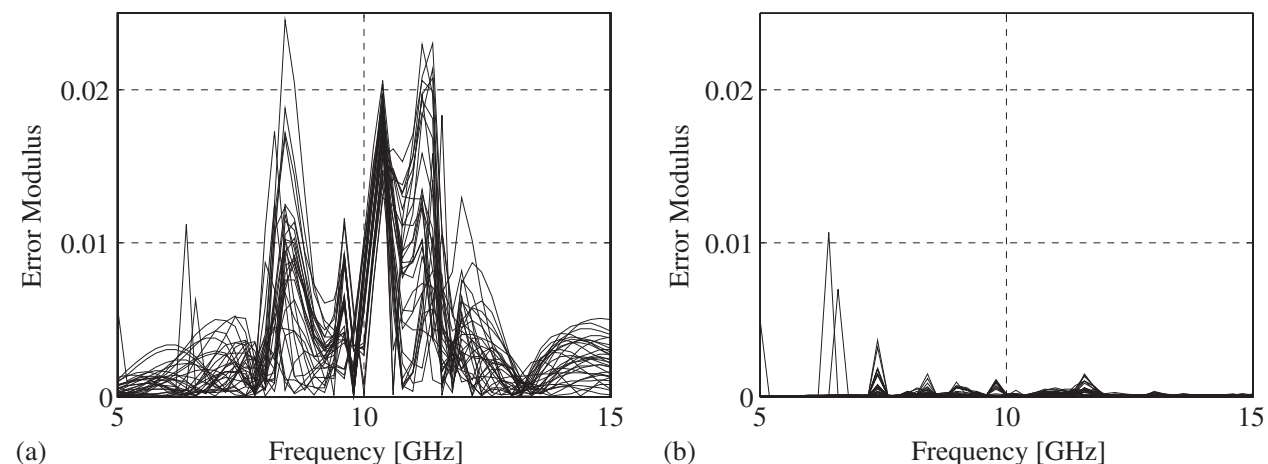

Figure 8. Test problem 3: error plots for (a) the SM-Standard and (b) SM-RBF surrogate models with base set $X_{B 3}$ (30 test points). 


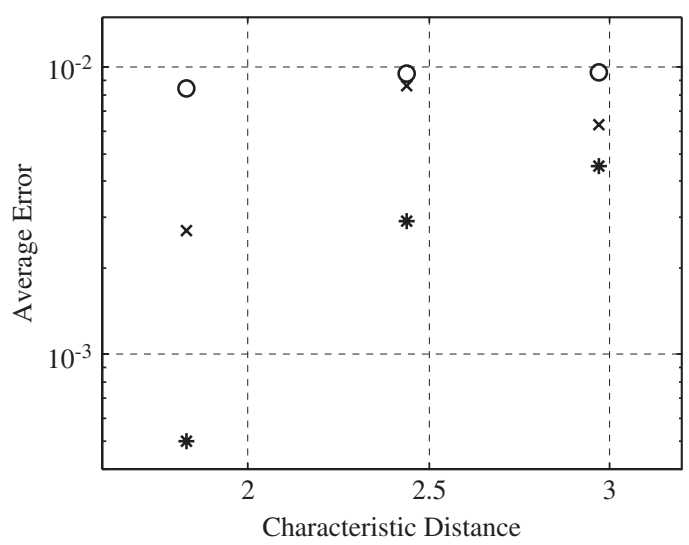

Figure 9. Test problem 1: average modeling error versus characteristic distance $\gamma$. Data for SM-Standard (o), SM-VWC (×), and SM-RBF (*).

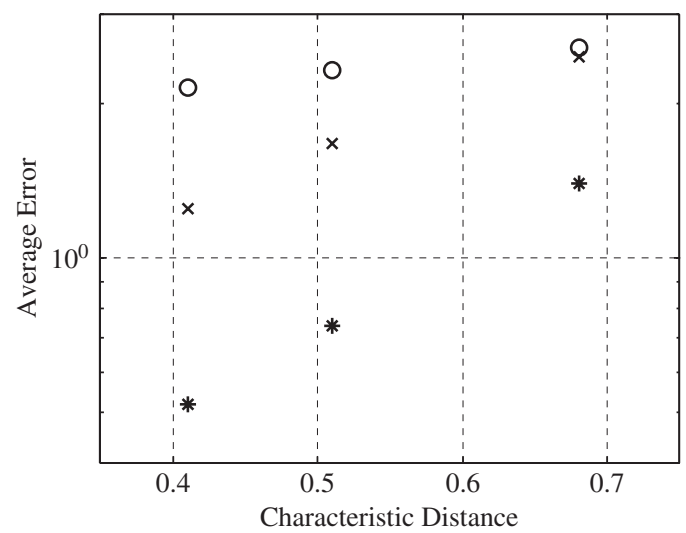

Figure 10. Test problem 2: average modeling error versus characteristic distance $\gamma$. Data for SM-Standard (o), SM-VWC (x), and SM-RBF (*).

weight coefficients. Moreover, the computational cost of the SM-RBF model is significantly lower than the cost of the SM-VWC model and almost the same as for the standard SM model. The reason is that the computational cost of SM-RBF and SM-Standard is virtually the same as the cost of the coarse model (after their parameters are established). The evaluation cost of the SM-VWC model is much higher because of the fact that each evaluation requires a separate parameter extraction, which typically amounts to hundreds of coarse model evaluations.

Table $\mathrm{V}$ gives the qualitative comparison of computational cost of the space mapping surrogate models. For the examples presented in this section, the evaluation time for the fine model is about $6 \mathrm{~min}$ (5 min, $45 \mathrm{~min}$ ) for Problem $1(2,3)$, while coarse model evaluation takes a couple of miliseconds for Problem 1 and about $1 \mathrm{~s}$ for Problems 2 and 3. Typical parameter extraction time is about $30 \mathrm{~s}$ for Problems 1 and 2, and up to several minutes for Problem 3. (For Problems 2 and 3, we use our inside-ADS optimization approach [34] in order to speed-up the parameter extraction process.) 


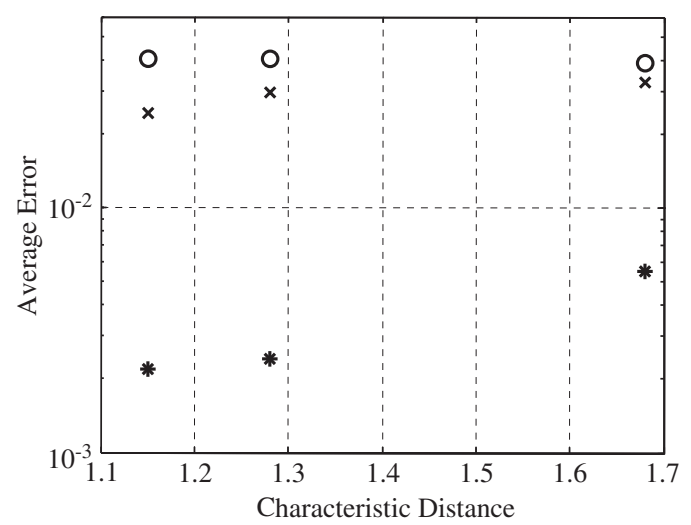

Figure 11. Test problem 3: average modeling error versus characteristic distance $\gamma$. Data for SM-Standard (o), SM-VWC (×), and SM-RBF (*).

Table V. Qualitative comparison of computational cost of evaluating the surrogate model.

\begin{tabular}{lcl}
\hline Surrogate model & Main sources of computational cost & Relative evaluation cost \\
\hline SM-Standard & Coarse model evaluation & Similar to coarse model \\
SM-VWC & Parameter extraction & Much higher than coarse model \\
SM-RBF & Coarse model evaluation & Similar to coarse model \\
\hline
\end{tabular}

Table VI. Comparison of modeling accuracy: space mapping versus direct RBF interpolation. Models developed for base set $X_{B 3}$.

\begin{tabular}{lcccc}
\hline & \multicolumn{3}{c}{ Average modeling error } \\
\cline { 2 - 4 } Test problem & SM-Standard & SM-VWC & SM-RBF & Direct RBF interpolation \\
\hline 1 & 0.0084 & 0.0027 & 0.0005 & 0.0142 \\
3 & 2.15 & 1.25 & 0.52 & 5.12 \\
& 0.0404 & 0.0280 & 0.0022 & 0.0054 \\
\hline
\end{tabular}

Note: Verification for 30 random test points.

Table VI gives a comparison of modeling accuracy between the space mapping surrogate models and direct RBF interpolation. The models were developed for test Problems 1, 2 and 3 described in Section 3.1 using the base set $X_{B 3}$. It is seen that the accuracy of the space mapping models is better than the accuracy of direct RBF interpolation in all cases except Problem 3 for SM-Standard and SM-VWC. For Problems 1 and 2, the performance of direct RBF interpolation is substantially worse than the performance of the space mapping approaches (regardless of the base set used, cf. Tables II-IV). This confirms that employing the physics-based coarse model, which is characteristic to space mapping, is essential to obtain good modeling accuracy, especially for large regions of interest as in Problems 1 and 2. Only in Problem 3, which is characterized by a relatively small region of interest, direct RBF interpolation beats the SM-Standard and SM-VWC, however it is outperformed by SM-RBF, which combines the features of space mapping and interpolation properties of radial basis functions. 


\section{APPLICATION EXAMPLES}

In this section, we present two examples of application of the space mapping surrogate models considered in the previous section to optimization and statistical analysis of microwave devices. The examples provide an additional comparison between the standard space mapping model and the space mapping model with radial basis function interpolation, verifying the robustness of the latter.

\subsection{Optimization of second-order tapped-line filter}

We use two space mapping surrogate models, the SM-Standard and SM-RBF, to optimize the second-order tapped-line filter described in Section 3. Surrogate models are obtained for the base set $X_{B 3}$ with parameters as described in Section 3.

The design specifications for the filter are: $\left|S_{21}\right| \leqslant-20 \mathrm{~dB}$ for $3.0 \mathrm{GHz} \leqslant \omega \leqslant 4.0 \mathrm{GHz},\left|S_{21}\right|$ $\geqslant-3 \mathrm{~dB}$ for $4.75 \mathrm{GHz} \leqslant \omega \leqslant 5.25 \mathrm{GHz}$, and $\left|S_{21}\right| \leqslant-20 \mathrm{~dB}$ for $6.0 \mathrm{GHz} \leqslant \omega \leqslant 7.0 \mathrm{GHz}$. The starting point for optimization is $\mathbf{x}^{0}=\left[\begin{array}{lll}6.977 & 0.060\end{array}\right]^{\mathrm{T}}$, which is the optimal solution of the coarse model. The fine model specification error at $\mathbf{x}^{0}$ is $+1.25 \mathrm{~dB}$.

Table VII shows the final solution as well as the specification error of the surrogate models and the fine model at corresponding surrogate model optima. Figures 12 and 13 show the fine and surrogate model responses at the surrogate model optimum for SM-Standard

Table VII. Optimization results of second-order tapped-line filter.

\begin{tabular}{|c|c|c|c|}
\hline Surrogate model & $\begin{array}{l}\text { Surrogate model } \\
\text { optimum }\end{array}$ & $\begin{array}{l}\text { Surrogate model specification } \\
\quad \text { error at optimum }(\mathrm{dB})\end{array}$ & $\begin{array}{l}\text { Fine model specification } \\
\text { error at optimum }(\mathrm{dB})\end{array}$ \\
\hline SM-Standard & {$\left[\begin{array}{lll}4.406 & 0.1936\end{array}\right]^{\mathrm{T}}$} & -0.78 & +1.06 \\
\hline SM-RBF & {$\left[\begin{array}{lll}5.893 & 0.0637\end{array}\right]^{\mathrm{T}}$} & -0.65 & -0.65 \\
\hline
\end{tabular}

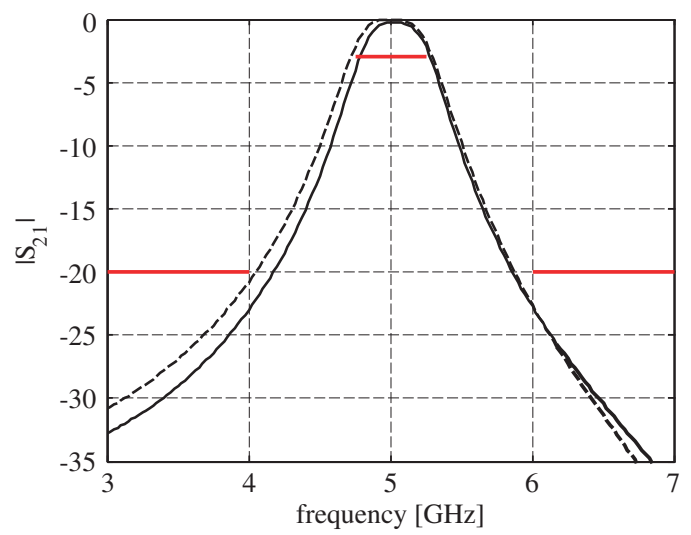

Figure 12. $\left|S_{21}\right|$ versus frequency for the second-order tapped-line filter at the optimal solution of the SM-Standard surrogate model: the fine model response (solid line) and the SM-Standard surrogate model response (dashed line). 
and SM-RBF, respectively. It is observed that the quality of the solution obtained with the SM-RBF model is much better than the quality of the solution obtained with the standard space mapping model. This is because of the better accuracy of the SM-RBF model, as indicated in Table II. Actually, the fine and SM-RBF surrogate model responses are indistinguishable in Figure 13.

\subsection{Statistical analysis of bandstop filter with open stubs}

We use two space mapping surrogate models, the SM-Standard and the SM-RBF, to perform statistical analysis of the bandstop filter with open stubs described in Section 3. For verification purposes, we also perform the analysis using the fine model. Surrogate models are obtained for the base set $X_{B 3}$ with parameters the same as in Section 3. The design specifications for the filter are: $\left|S_{21}\right| \geqslant 0.95$ for $5.0 \mathrm{GHz} \leqslant \omega \leqslant 8.0 \mathrm{GHz},\left|S_{21}\right| \leqslant 0.05$ for $9.4 \mathrm{GHz} \leqslant \omega \leqslant 10.6 \mathrm{GHz}$, and $\left|S_{21}\right|$ $\geqslant 0.95$ for $12.0 \mathrm{GHz} \leqslant \omega \leqslant 15.0 \mathrm{GHz}$. We perform statistical analysis around the fine model optimal solution with respect to these specifications, which is $\mathbf{x}=\left[\begin{array}{lllll}5.6 & 10.4 & 119.2 & 118 & 112\end{array}\right]^{\mathrm{T}} \mathrm{mil}$ (the reference point used in Section 3 for Problem 3) with a maximum deviation of the design variables of 0.04 mil for $W_{1}, W_{2}$ and 2 mil for $L_{0}, L_{1}, L_{2}$, which corresponds to the region size $\boldsymbol{\delta}$ used for Problem 3 in Section 3.

The results of statistical analysis are used to estimate the yield, i.e. the percentage of points for which the design specifications are satisfied. Yield estimation obtained using the SM-Standard

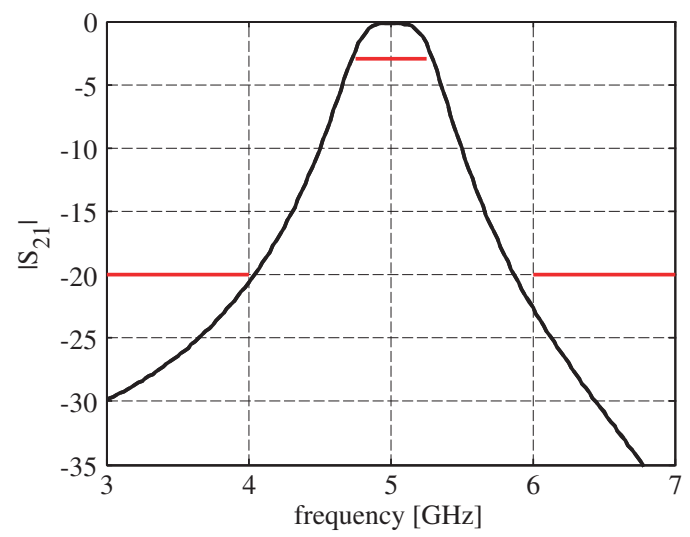

Figure 13. $\left|S_{21}\right|$ versus frequency for the second-order tapped-line filter at the optimal solution of the SM-RBF surrogate model: the fine model response (solid line) and the

SM-RBF surrogate model response (dashed line).

Table VIII. Statistical analysis of bandstop filter with open stubs.

\begin{tabular}{lc}
\hline Model used in statistical analysis & Yield estimation (\%) \\
\hline SM-Standard & 69.5 \\
SM-RBF & 63.0 \\
Fine model & 63.5 \\
\hline
\end{tabular}




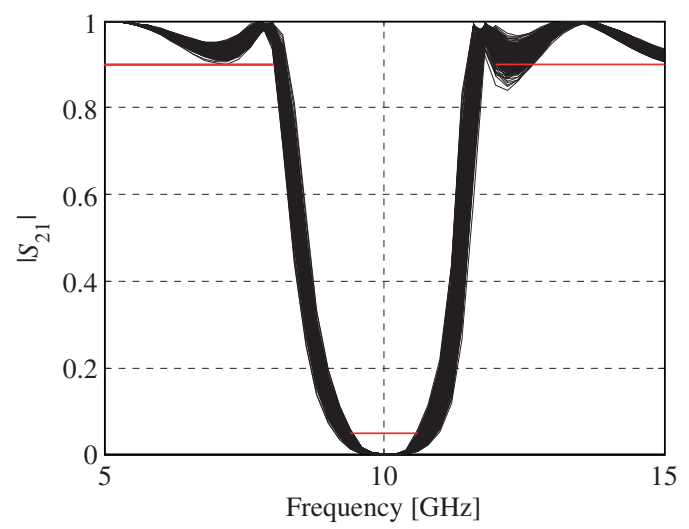

Figure 14. Bandstop filter with open stubs: a statistical analysis at the fine model optimal solution $\mathbf{x}=\left[\begin{array}{lllll}5.6 & 10.4 & 119.2 & 118 & 112\end{array}\right]^{\mathrm{T}}$ mil using the SM-Standard surrogate model. Estimated yield $69.5 \%$; 200 random test points; maximum deviation 0.04 mil for $W_{1}, W_{2}$, and 2 mil for $L_{0}, L_{1}, L_{2}$.

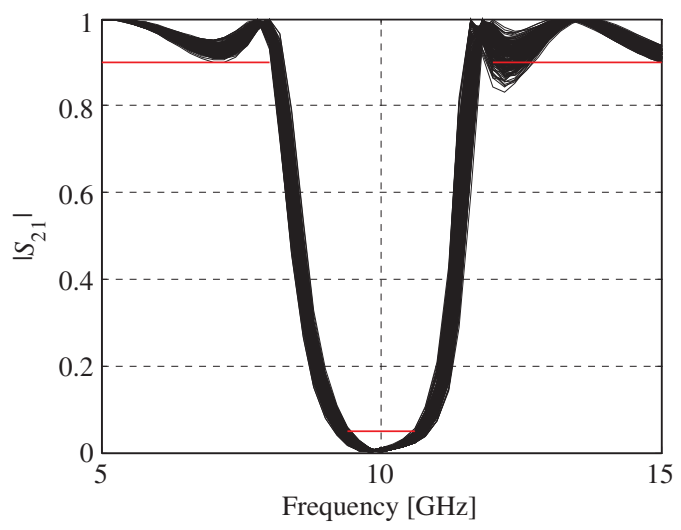

Figure 15. Bandstop filter with open stubs: a statistical analysis at the fine model optimal solution $\mathbf{x}=\left[\begin{array}{lllll}5.6 & 10.4 & 119.2 & 118 & 112\end{array}\right]^{\mathrm{T}}$ mil using the SM-RBF model. Estimated yield $63.0 \% ; 200$ random test points; maximum deviation 0.04 mil for $W_{1}$, $W_{2}$, and 2 mil for $L_{0}, L_{1}, L_{2}$.

and SM-RBF surrogate models as well as the fine model are given in Table VIII. $\left|S_{21}\right|$ plots for all the models are shown in Figures 14-16. It is observed that the SM-RBF model gives a better prediction of yield than the standard SM model although the prediction given by SM-Standard is also decent. Note that the plot for the SM-Standard model (Figure 14) is visually different from the plot for the fine model (Figure 16), however, the plot for the SM-RBF model (Figure 15 ) is visually indistinguishable from the plot for the fine model. It should be noted that statistical analysis performed using the fine model took almost a week because of the computational cost of evaluating the model (about $45 \mathrm{~min}$ per evaluation). Analysis using the space mapping surrogate models takes less than a few seconds when using the multi-point evaluation capability of the coarse model simulator (cf. [34]). 


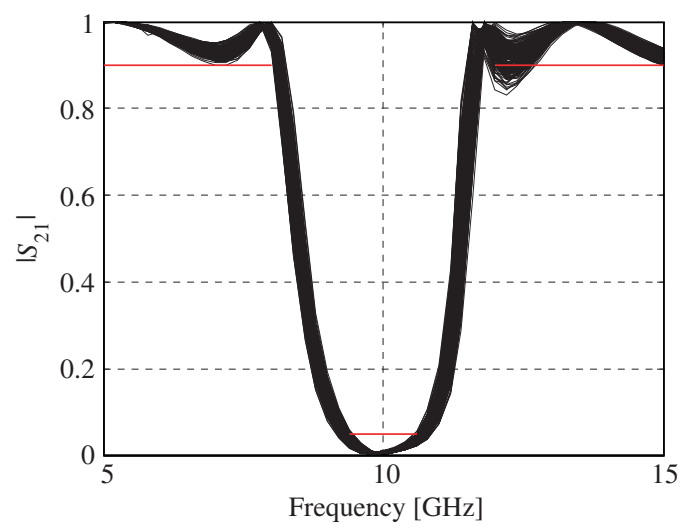

Figure 16. Bandstop filter with open stubs: a statistical analysis at the fine model optimal solution $\mathbf{x}=\left[\begin{array}{lllll}5.6 & 10.4 & 119.2 & 118 & 112\end{array}\right]^{\mathrm{T}}$ mil using the fine model. Estimated yield $63.5 \%$; 200 random test points; maximum deviation 0.04 mil for $W_{1}, W_{2}$, and 2 mil for $L_{0}, L_{1}, L_{2}$.

\section{CONCLUSION}

A review of recent developments in space mapping modeling methodology is presented. A comparison of several modeling techniques is given, including a novel modeling technique that combines space mapping with radial basis function interpolation. It is shown that this combination allows, without compromising the computational cost, the reduction of the modeling error to a level not attainable for any other space mapping techniques thus far. Application examples demonstrate the robustness of the method.

\section{ACKNOWLEDGEMENTS}

The authors thank Sonnet Software, Inc., Syracuse, NY, for $\boldsymbol{e m}^{\mathrm{TM}}$ and Agilent Technologies, Santa Rosa, CA, for making ADS available. This work was supported in part by the Natural Sciences and Engineering Research Council of Canada under Grants OGP0007239 and STPGP336760.

\section{REFERENCES}

1. Bandler JW, Biernacki RM, Chen SH, Grobelny PA, Hemmers RH. Space mapping technique for electromagnetic optimization. IEEE Transactions on Microwave Theory and Techniques 1994; 4(12):536-544.

2. Bandler JW, Cheng QS, Dakroury SA, Mohamed AS, Bakr MH, Madsen K, Sondergaard J. Space mapping: the state of the art. IEEE Transactions on Microwave Theory and Techniques 2004; 52(1):337-361.

3. Koziel S, Bandler JW, Madsen K. A space mapping framework for engineering optimization: theory and implementation. IEEE Transactions on Microwave Theory and Techniques 2006; 54(10):3721-3730.

4. Echeverria D, Hemker PW. Space mapping and defect correction. CMAM the International Mathematical Journal Computational Methods in Applied Mathematics 2005; 5(2):107-136.

5. Choi H-S, Kim DH, Park IH, Hahn SY. A new design technique of magnetic systems using space mapping algorithm. IEEE Transactions on Magnetics 2001; 37(5):3627-3630.

6. Ismail MA, Smith D, Panariello A, Wang Y, Yu M. EM-based design of large-scale dielectric-resonator filters and multiplexers by space mapping. IEEE Transactions on Microwave Theory and Techniques 2004; 52(1):386-392.

7. Amari S, LeDrew C, Menzel W. Space-mapping optimization of planar coupled-resonator microwave filters. IEEE Transactions on Microwave Theory and Techniques 2006; 54(5):2153-2159. 
8. Rayas-Sánchez JE, Gutiérrez-Ayala V. EM-based Monte Carlo analysis and yield prediction of microwave circuits using linear-input neural-output space mapping. IEEE Transactions on Microwave Theory and Techniques 2006; 54(12):4528-4537.

9. Bandler JW, Georgieva N, Ismail MA, Rayas-Sánchez JE, Zhang QJ. A generalized space mapping tableau approach to device modeling. IEEE Transactions on Microwave Theory and Techniques 2001; 49(1):67-79.

10. Koziel S, Bandler JW, Mohamed AS, Madsen K. Enhanced surrogate models for statistical design exploiting space mapping technology. IEEE MTT-S International Microwave Symposium Digest, Long Beach, CA, June 2005; 1609-1612.

11. Bandler JW, Cheng QS, Koziel S. Simplified space mapping approach to enhancement of microwave device models. International Journal of $R F$ and Microwave Computer-Aided Engineering 2006; 16(5):518-535.

12. Rautio JC. A space mapped model of thick, tightly coupled conductors for planar electromagnetic analysis. IEEE Microwave Magazine 2004; 5(3):62-72.

13. Koziel S, Bandler JW. Space-mapping-based modeling utilizing parameter extraction with variable weight coefficients and a data base. IEEE MTT-S International Microwave Symposium Digest, San Francisco, CA, June 2006; 1763-1766.

14. Koziel S, Bandler JW, Madsen K. Theoretical justification of space-mapping-based modeling utilizing a data base and on-demand parameter extraction. IEEE Transactions on Microwave Theory and Techniques 2006; 54(12):4316-4322.

15. Koziel S, Bandler JW. Microwave device modeling utilizing parameter extraction and radial basis functions. IEEE MTT-S International Microwave Symposium Digest, Honolulu, Hawaii, June 2007; 799-802.

16. Zhang L, Xu JJ, Yagoub M, Ding RT, Zhang QJ. Neuro-space mapping technique for nonlinear device modeling and large signal simulation. IEEE MTT-S International Microwave Symposium Digest, Philadelphia, PA, June 2003; $173-176$.

17. Devabhaktuni VK, Chattaraj B, Yagoub MCE, Zhang Q-J. Advanced microwave modeling framework exploiting automatic model generation, knowledge neural networks, and space mapping. IEEE Transactions on Microwave Theory and Techniques 2003; 51(7):1822-1833.

18. Zhang L, Xu J, Yagoub MCE, Ding R, Zhang Q-J. Efficient analytical formulation and sensitivity analysis of neurospace mapping for nonlinear microwave device modeling. IEEE Transactions on Microwave Theory and Techniques 2005; 53(9):2752-2767.

19. Burrascano P, Dionigi M, Fancelli C, Mongiardo M. A neural network model for CAD and optimization of microwave filters. IEEE MTT-S International Microwave Symposium Digest, Baltimore, MD, 1998; $13-16$.

20. Peik SF, Mansour RR, Chow YL. Multidimensional Cauchy method and adaptive sampling for an accurate microwave circuit modeling. IEEE Transactions on Microwave Theory and Techniques 1998; 46(12):2364-2371.

21. Simpson TW, Peplinski J, Koch PN, Allen JK. Metamodels for computer-based engineering design: survey and recommendations. Engineering with Computers 2001; 17(2):129-150.

22. Queipo NV, Haftka RT, Shyy W, Goel T, Vaidynathan R, Tucker PK. Surrogate-based analysis and optimization. Progress in Aerospace Sciences 2005; 41(1):1-28.

23. Mullur AA, Messac A. Metamodeling using extended radial basis functions: a comparative approach. Engineering with Computers 2006; 21(3):203-217.

24. Simpson TW, Maurey TM, Korte JJ, Mistree F. Kriging models for global approximation in simulation-based multidisciplinary design optimization. AIAA Journal 2001; 39(12):2233-2241.

25. Powell MJD. Radial basis functions for multivariate interpolation: a review. In Algorithms for Approximation, Mason JC, Cox MG (eds). Clarendon Press: Oxford, 1987.

26. Buhmann MD, Ablowitz MJ. Radial Basis Functions: Theory and Implementations. Cambridge University Press: Cambridge, U.K., 2003.

27. Bakr MH, Bandler JW, Madsen K, Søndergaard J. An introduction to the space mapping technique. Optimization Engineering 2001; 2(4):369-384.

28. Sonnet Software, Inc. $\mathrm{em}^{\mathrm{TM}}$ Version 10.53, Sonnet Software, Inc., North Syracuse, NY, U.S.A.

29. Kirschning M, Jansen RH, Koster NHL. Measurement and computer-aided modeling of microstrip discontinuities by an improved resonator method. IEEE MTT-S International Microwave Symposium Digest, Boston, MA, May 1983; 495-497.

30. Manchec A, Quendo C, Favennec J-F, Rius E, Person C. Synthesis of capacitive-coupled dual-behavior resonator (CCDBR) filters. IEEE Transactions on Microwave Theory and Techniques 2006; 54(6):2346-2355.

31. EM Software \& Systems-S.A. (Pty) Ltd. FEKO ${ }^{\circledR}$ User's Manual, EM Software \& Systems-S.A. (Pty) Ltd, Stellenbosch, South Africa, June 2004, http://www.feko.info

32. Agilent ADS. Version 2003C, Agilent Technologies, Santa Rosa, CA, 2003.

33. Bakr MH, Bandler JW, Ismail MA, Rayas-Sanchez JE, Zhang QJ. Neural space-mapping optimization for EM-based design. IEEE Transactions on Microwave Theory and Techniques 2000; 48(12):2307-2315.

34. Koziel S, Cheng QS, Bandler JW. Improving efficiency of space mapping optimization of microwave structures and devices. IEEE MTT-S International Microwave Symposium Digest, Honolulu, Hawaii, June 2007; 1995-1998. 


\section{AUTHORS’ BIOGRAPHIES}

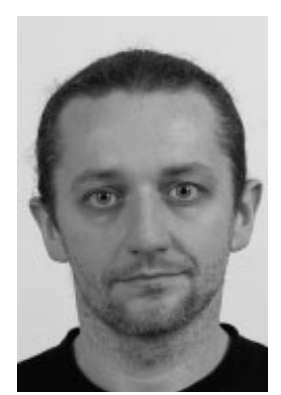

Slawomir Koziel received the MSc and $\mathrm{PhD}$ degrees in electronic engineering from Gdansk University of Technology, Poland, in 1995 and 2000, respectively. He also received the MSc degrees in theoretical physics and in mathematics, in 2000 and 2002, respectively, as well as the $\mathrm{PhD}$ in mathematics in 2003, from the University of Gdansk, Poland. He is currently a Research Associate in the Department of Electrical and Computer Engineering, McMaster University, Canada. He has published more than 100 papers. His research interests include space mapping, circuit theory, analog signal processing, evolutionary computation and numerical analysis.

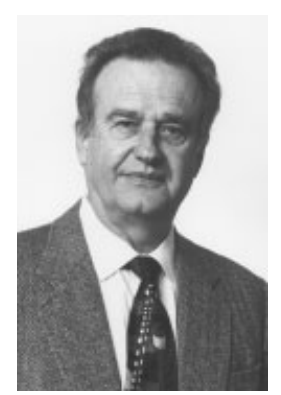

John W. Bandler studied at Imperial College of Science and Technology and received the BSc(Eng.), PhD, and DSc(Eng.) degrees from the University of London, England, in 1963, 1967, and 1976, respectively.

He joined Mullard Research Laboratories, Redhill, Surrey, England in 1966. From 1967 to 1969 he was a Postdoctorate Fellow and Sessional Lecturer at the University of Manitoba, Winnipeg, Canada. He joined McMaster University, Canada, in 1969. He has served as Chairman of the Department of Electrical Engineering and Dean of the Faculty of Engineering. He is now Professor Emeritus. He was President of Optimization Systems Associates Inc., which he founded in 1983, until November 20, 1997, the date of acquisition by Hewlett-Packard Company. He is President of Bandler Corporation, which he founded in 1997.

He has served as associate editor, guest editor, and on the editorial boards of various engineering journals and organizations. He has served as a member and chair of the IEEE MTT Society MTT-1 Technical Committee on Computer-Aided Design. He has published more than 400 papers.

$\mathrm{He}$ is a Fellow of the Canadian Academy of Engineering, a Fellow of the Royal Society of Canada, a Fellow of the Institution of Electrical Engineers (Great Britain), a Fellow of the Engineering Institute of Canada, a Member of the Association of Professional Engineers of the Province of Ontario (Canada) and a Member of the MIT Electromagnetics Academy. He received the Automatic Radio Frequency Techniques Group (ARFTG) Automated Measurements Career Award in 1994 and the IEEE MTT-S Microwave Application Award in 2004. 